\title{
EchoGéo
}

45 | 2018

Déclinaisons géographiques du changement social en Iran

\section{Niloufar Niksar. Pour une architecture du discernement à Téhéran}

Entretien réalisé à distance le 6 septembre 2018 et traduit du persan par Amin Moghadam

\section{Niloufar Niksar et Amin Moghadam}

\section{(2) OpenEdition}

\section{Journals}

Édition électronique

URL : https://journals.openedition.org/echogeo/16023

DOI : $10.4000 /$ echogeo.16023

ISSN : 1963-1197

Éditeur

Pôle de recherche pour l'organisation et la diffusion de l'information géographique (CNRS UMR 8586)

Référence électronique

Niloufar Niksar et Amin Moghadam, « Niloufar Niksar. Pour une architecture du discernement à

Téhéran », EchoGéo [En ligne], 45 | 2018, mis en ligne le 05 novembre 2018, consulté le 10 août 2021.

URL : http://journals.openedition.org/echogeo/16023 ; DOI : https://doi.org/10.4000/echogeo.16023

Ce document a été généré automatiquement le 10 août 2021.

EchoGéo est mis à disposition selon les termes de la licence Creative Commons Attribution - Pas d'Utilisation Commerciale - Pas de Modification 4.0 International (CC BY-NC-ND) 


\section{Niloufar Niksar. Pour une architecture du discernement à Téhéran}

Entretien réalisé à distance le 6 septembre 2018 et traduit du persan par Amin Moghadam

Niloufar Niksar et Amin Moghadam

1 La question du logement a été au centre des débats politiques et économiques de l'Iran contemporain. L'instabilité économique, le caractère rentier de l'économie iranienne et le taux élevé de liquidités ont souvent transformé le marché immobilier en un lieu idéal pour les investissements de capitaux. Alors que selon le recensement de 2016, il y aurait un déficit de 1,39 million de logements par rapport à la demande, les pratiques spéculatives ont laissé plus de 2,5 millions ( $30 \%$ de plus qu'en 2011) d'unités d'habitation - immobilier de luxe le plus souvent - vides qui ne correspondent pas à la demande de logement. En même temps, $30 \%$ de la population urbaine du pays habite dans les tissus anciens des villes ou dans des espaces informels. Dans un contexte d'économie de marché et de connivence avec l'État, les possibilités d'enrichissement par le biais d'investissements dans les projets de rénovation urbaine ont abouti à des phénomènes de gentrification, connus ailleurs dans le monde, et au déplacement des populations vers des zones plus éloignées

L'expérience de Niloufar Niksar reflète quelques enjeux majeurs relatifs au logement et à la rénovation urbaine en Iran qui se traduisent dans l'exercice du métier d'architecte et d'urbaniste par des formes d'adaptation et de questionnement vis-à-vis du système dominant du marché.

2 - Amin Moghadam (AM). Je sais que vous avez participé dans les dernières années à de nombreux projets architecturaux et urbains en Iran. Pourriez-vous revenir sur ces expériences et nous donner une vue globale des types de projets auxquels vous avez participé mais aussi sur votre parcours éducatif et professionnel ?

3 - Niloufar Niksar (NN). J'ai été diplômée d'architecture en 2002 en Iran, à l'Université d'Azad, et j'ai commencé ma carrière dans des projets à grande échelle, notamment des 
projets industriels. J'étais particulièrement impliquée entre 2000-2002 dans un projet industriel à Assaluyeyh, dans le sud iranien, où se trouve le plus grand projet gazier du pays. Ceci est devenu également mon projet de fin d'étude en architecture. Je me posais des questions pendant cette expérience sur l'impact du capital investi dans cet environnement sur les populations locales et la manière dont je pourrais, en tant qu'architecte, créer des espaces pour toutes les populations présentes dans les villages environnant ce projet industriel de grande ampleur et d'importance nationale, sachant qu'il avait attiré des populations d'origines diverses. Cette expérience a constitué ma première sensibilisation aux questions sociales qui peuvent se poser au travail de l'architecte.

4 - AM. Dans vos écrits et vos travaux ${ }^{1}$, vous évoquez souvent la question de la participation et la reconnaissance du rôle des usagers dans l'espace. Comment voyezvous ces questions dans votre travail et plus globalement comment pensez-vous que ces questions sont abordées dans l'environnement de travail, dans votre milieu professionnel milieu professionnel et plus généralement en Iran d'aujourd'hui?

5 - NN. Quelques années après l'expérience d'Assaluyeh, une photographe allemande, Beatrice Minda s'est rendue en Iran ; elle voulait réaliser un ouvrage de photos sur les intérieurs iraniens en faisant parler des personnes qui y habitaient, par exemple dans les appartements. Elle m'a demandé de l'aider. Elle publié son travail sous forme d'un ouvrage intitulé Iran. Interrupted ${ }^{2}$. Lors de ce projet, je me suis rendue compte que les habitants ne savaient pas forcément parler de leur espace. Le dialogue qui s'est établi entre moi et Beatrice m'a permis de me poser la question de comment, en tant qu'architecte, je peux définir l'espace en fonction des pratiques et du vécu et non pas seulement par la morphologie. En Iran, cette approche n'est pas vraiment prise en compte par les maîtres d'ouvrage. Comme partout d'ailleurs, c'est le profit financier qui les intéresse en premier lieu.

6 Mais moi, en tant qu'architecte, je me place entre le maitre d'ouvrage et les usagers et j'ai commencé aussi à m'intéresser à la manière dont je pouvais prendre en compte dans mon travail le point de vue des habitants et des usagers. Comme il n'y a pas de formation à cette approche ni d'intérêt pour de la part des maitres d'ouvrage dans ce métier, je me suis sentie obligée, dans mon travail, de deviner la typologie des personnes qui vont vivre dans les appartements que je conçois. J'essaie de le voir à partir de la manière dont ils s'habillent ou de leur manière de parler et j'essaie de comprendre par exemple s'ils viennent d'une famille religieuse ou pas.

7 Ce dernier critère est très important bien sûr car il affecte la conception des espaces ouverts et fermés. Par exemple, il y a encore quelques années, les balcons n'avaient pas de justification économique dans les projets architecturaux et les maîtres d'ouvrage n'étaient pas toujours convaincus de la nécessité de les mettre en place. Mais ce point de vue a changé. J'aime personnellement des espaces ouverts et je me bats parfois pour les faire passer même s'ils peuvent avoir une fonction différente de celles qu'on connait. Par exemple, un maitre d'ouvrage me disait « Mais si tu fais des balcons pour ces gens traditionnels, ils vont mettre leurs pickles et leur produits alimentaires sur le balcon» et je lui ai répondu «Et alors? Tout le monde ne doit pas mettre une belle table ou un beau pot de fleur pour profiter du balcon. Cela veut dire qu'ils s'intéressent à la cuisine et pourquoi n'utiliseraient-ils pas leur balcon à cette fin? ?. 
Illustration 1 - Le projet de logement numéro 12 par Niloufar Niksar, 2012-2014.

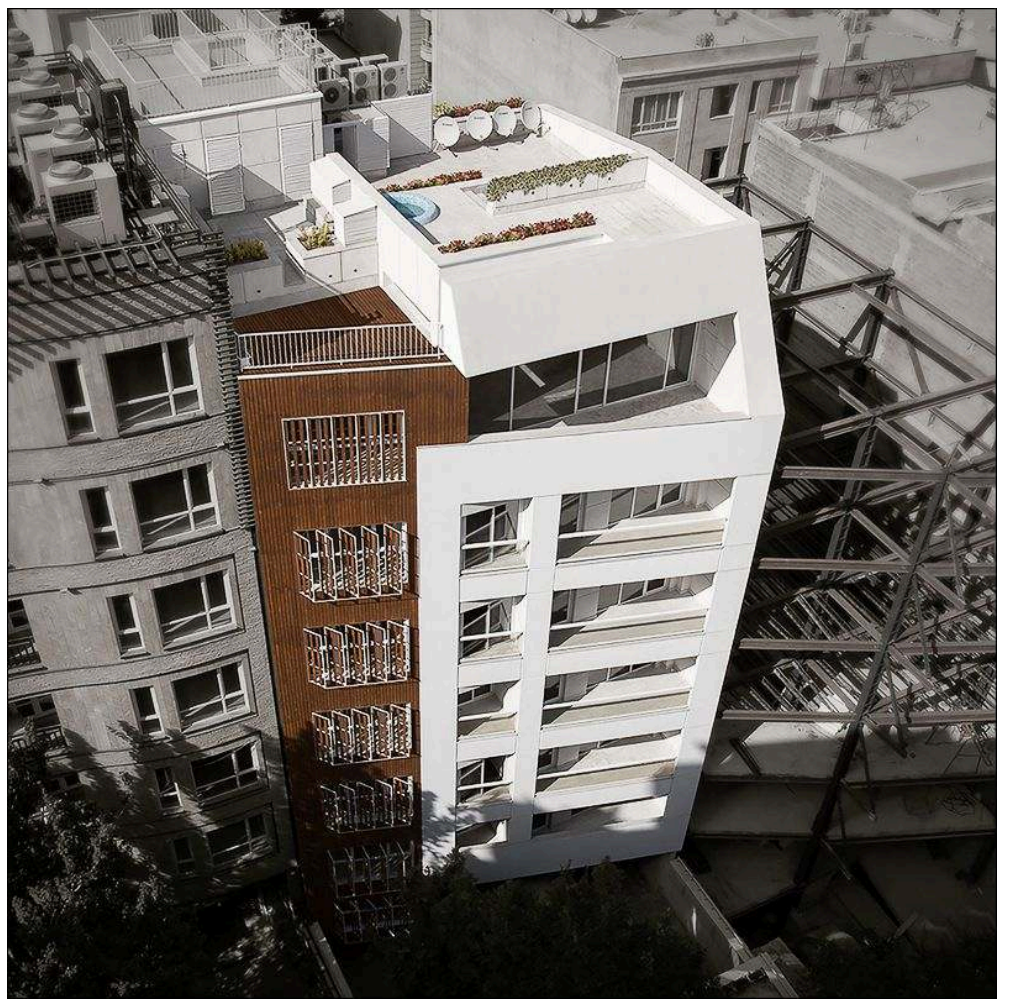

Auteur: Sh. Mokhaber.

Parfois je demande à visiter l'apparemment après l'avoir délivré. Je prends du plaisir à voir si j'ai bien deviné la manière dont les usagers vont aménager l'appartement ou pas, s'ils ont bien gardé l'axe que j'ai pensé être le meilleur ou bien s'ils l'ont complètement changé à leur manière. Je regarde aussi parfois à travers les rideaux des immeubles sur lesquels j'ai travaillé pour voir qui y habite et comment ils l'ont aménagé.

9 En 2016, le ministère iranien de logement et de développement urbain a organisé une compétition pour la sélection d'un projet que le pays allait présenter à biennale de Venise. J'ai décidé d'y participer. J'ai été très influencée dans mon parcours par les idées de Majid Rahnama ${ }^{3}$ qui portent principalement sur le rôle de la participation, c'est ce qui a donné lieu à un projet d'installation. Notre stand portait sur le droit à la participation et s'appuyant sur la phrase de Rahnama «Écouter, vivre et apprendre ». Mon projet a été dans les huit premiers mais, finalement, il n'a pas été sélectionné. C'est avec cette vision que j'aime exercer mon métier, c'est-à-dire mener des travaux de terrain et aller chez les habitants. 
Illustration 2 - «Écouter, vivre et apprendre ». Proposition de Niloufar Niksar pour le pavillon d'Iran à la Biennale de Venise 2016.

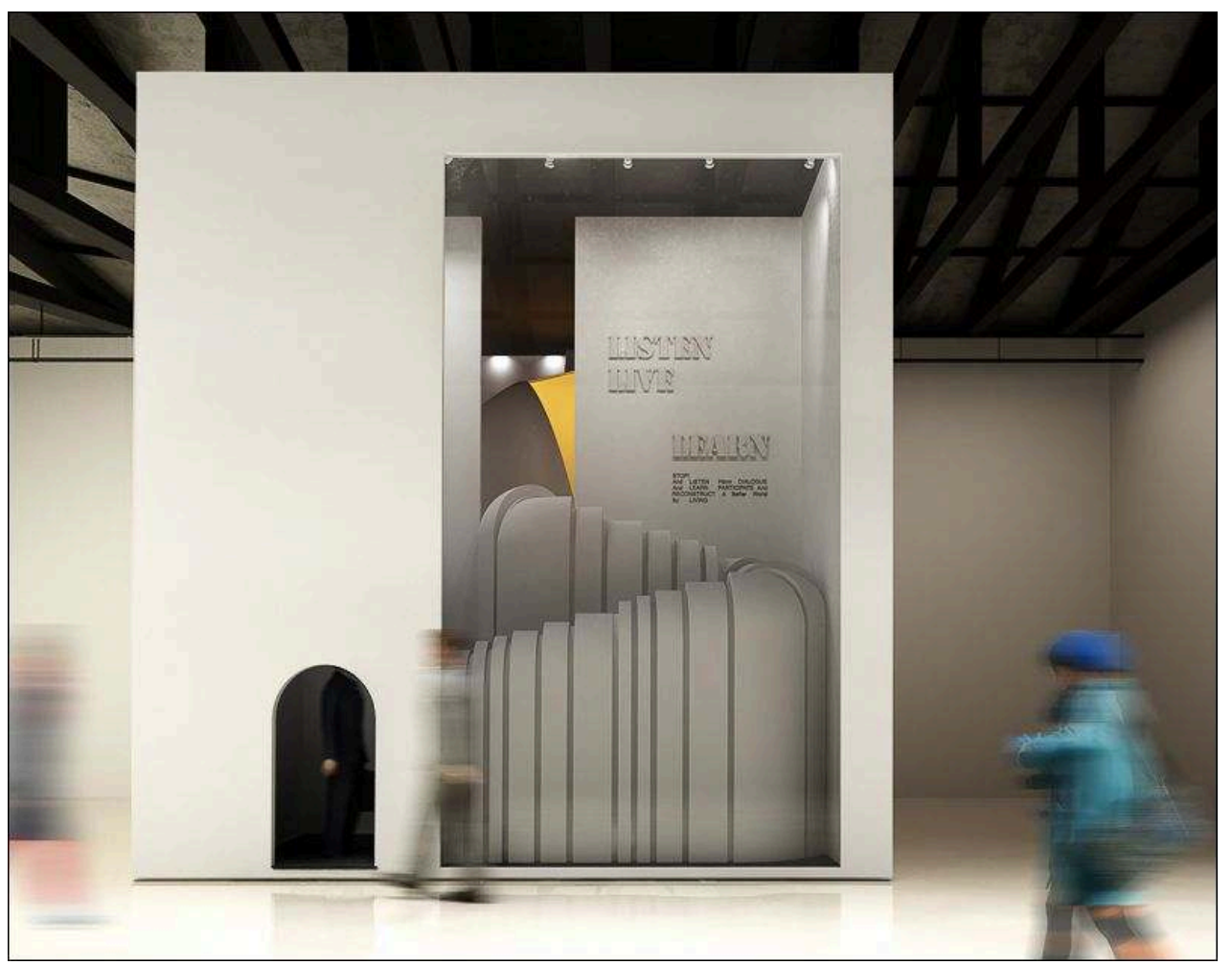

Proposition soumise au ministère des Transports et du développement urbain iranien

Auteur : Niloufar Niksar.

Cet apprentissage pour les architectes nous permet de dire que les gens peuvent parler de leur espace, tout le monde n'est pas capable de parler de son propre espace, c'est un apprentissage.

11 - AM. Dans vos écrits, vous exprimez souvent votre opposition au système capitaliste qui règne en Iran et en particulier dans les projets urbains et architecturaux. Vous questionnez le rôle, voire la contribution explicite des architectes à ce système. Avec ce regard et ce point de vue, comment arrivez-vous à maintenir votre identité d'architecte ou votre "agency", pour reprendre le terme que vous employez souvent dans les projets auxquels vous participez, et dans le contexte actuel de l'Iran ?

12 - NN. L'instabilité du marché, en plus de la culture professionnelle, affectent beaucoup nos manières de faire de l'architecture. Cette situation a créé une culture de la «toute vitesse » et bizarrement les usagers ont aussi adopté cette culture. En même temps, les matériaux de certains projets sont importés et parfois par des mafias qui bénéficient de leur connexion avec les marchés extérieurs pour l'importation des matériaux, ce qui aussi augmente le prix des appartements. Pour l'architecte que je suis, c'est toujours plus agréable de travailler avec de meilleurs matériaux mais en même temps je sais que ce type de projet ne s'adresse pas à tout le monde et qu'il y a d'énormes spéculations autour des projets architecturaux.

13 L'architecture est très politique dans les grands projets mais, très rarement, les personnes concernées, même pour les projets de logements sociaux, prennent en compte les questions sociales. Par exemple, j'étais impliquée dans une étude pour les logements sociaux de $\mathrm{Mehr}^{4}$ et en fait on nous demandait juste de dupliquer un modèle 
d'appartement avec une cuisine à l'américaine, deux chambres etc. De la même manière, les usagers aussi cherchent leurs modèles dans les séries télévisées et ce qu'ils voient dans les journaux, sans nécessairement prendre en compte les spécificités de leur environnement de vie. Ils veulent juste reproduire ce qu'ils voient chez les autres sans penser à leur propre condition.

Dans mon travail d'architecte, je dois adopter « une politique de jugeote » : auprès de l'investisseur, je dois dire «oui, toutes nos propositions conviennent à vos investissements ", on peut difficilement parler de participation avec les investisseurs. Compte tenu de la capacité de l'architecture à pouvoir faire circuler des capitaux énormes en Iran, il est presque impossible de parler des considérations sociales. En même temps, on doit faire attention aux usagers. Je dis toujours qu'un bon architecte est un " architecte qui a de la jugeote ", je veux dire par là un architecte qui peut faire un pont entre les différents acteurs qui ont souvent ont des intérêts divergents. Certains, plus radicaux, pensent que les architectes ne devraient pas accepter certains projets mais on ne peut pas généraliser cela. C'est possible de refuser certains projets mais si on veut s'opposer à tout type d'investissement alors on doit arrêter de faire de l'architecture. C'est mon métier et je dois finalement accepter certains projets.

Parler dans la sphère théorique est attirant mais en réalité je dois exercer un métier. Je me pose tous les jours la question de savoir comment est-ce qu'on peut inclure des idées théoriques dans mes projets architecturaux mais je sais que ne rien faire n'est pas une solution. Peut-être qu'un jour j'arriverai à cette conclusion de ne plus travailler mais pour le moment j'ai appris à parler dans un langage économique avec l'investisseur et à prendre en compte en même temps les intérêts des usagers. J'essaie dans mon discours d'établir une convergence entre les deux groupes d'acteurs. C'est un travail très politique et parfois cela n'a rien à voir avec l'architecture.

- AM. Et si on parlait maintenant des projets urbains dans lesquels vous avez été impliquée. Vous avez fait les deux types d'expériences de projets, urbains et architecturaux. Par exemple dans le projet «Oudlajan", un espace pour le changement ", vous posez encore une fois la question de la participation. Pourriez-vous parler un peu de ce projet?

17 - NN. Le projet d'Oudlajan a été conçu dans le cadre d'une compétition. L'origine de ce projet remonte à 2008 l'année pendant laquelle on travaillait avec Ayat Najafi ${ }^{6}$ et mon mari sur une pièce de théâtre qui s'appelait « Tehran, Banou » (Madame Téhéran), dont le récit portait sur une femme juive et musicienne qui habitait dans le quartier d'Oudlajan et avait des histoires dans ce quartier. L'histoire se déroulait pendant la deuxième guerre mondiale, au moment de l'arrivée des étrangers en Iran durant la guerre. A cette époque, le centre de Téhéran, comme l'origine historique du Téhéran actuel, m'intéressait beaucoup. On écrivait ensemble le texte de cette pièce et on la changeait en fonction aussi de nos données historiques sur la ville. Pourria, mon mari, a commencé à faire des vidéos dans ce quartier. Le quartier changeait très rapidement et on parlait avec les habitants; on leur demandait toujours « Vous savez pourquoi ils ont détruit telle partie de la ville? " et souvent on recevait des réponses contradictoires comme « on a entendu que les autorités de la ville veulent construire une périphérie pour la ville de Téhéran à travers le quartier Pamenar ».

On se rendait compte que les habitants n'étaient absolument pas au courant des projets et ils étaient en même temps mécontents de vivre dans un chantier permanent. Les autorités de la ville de Téhéran ont vraiment créé une rupture dans un tissu ancien de 
la ville, on aurait dit qu'ils avaient retiré le cœur vivant du quartier. C'était un projet très politique. Quand il y a eu la compétition sur ce quartier, on a décidé de faire une proposition et notre idée consistait surtout à inclure nos données qualitatives du quartier dans le projet. En même temps, des questions telles que la gentrification commençaient à avoir un certain succès dans les milieux professionnels travaillant sur la ville. Il s'agit du quartier le plus ancien de Téhéran, qui était traditionnellement habité par une classe sociale plutôt moyenne, des gens ordinaires et non pas par la bourgeoisie qui peuplait les anciens quartiers de la ville. Une partie travaillait dans le grand bazar de Téhéran. Aussi pendant la guerre avec l'Irak (1980-1988), le gouvernement avait accueilli de nombreux rescapés de guerre dans ces quartiers et cela avait changé la démographie et la composition sociale de ce quartier. Le conflit qui s'est alors fait jour était entre les gens du bazar qui représentaient la partie conservatrice du pays, les technocrates qui avaient été formés plus récemment dans la période postrévolutionnaire et les personnes dont l'intérêt consiste à gentrifier des quartiers comme Oudlajan. Dans tout cela, les habitants n'avaient aucun rôle.

Illustration 3 « Notre Oudlajan, un espace de participation et de changement », 2016

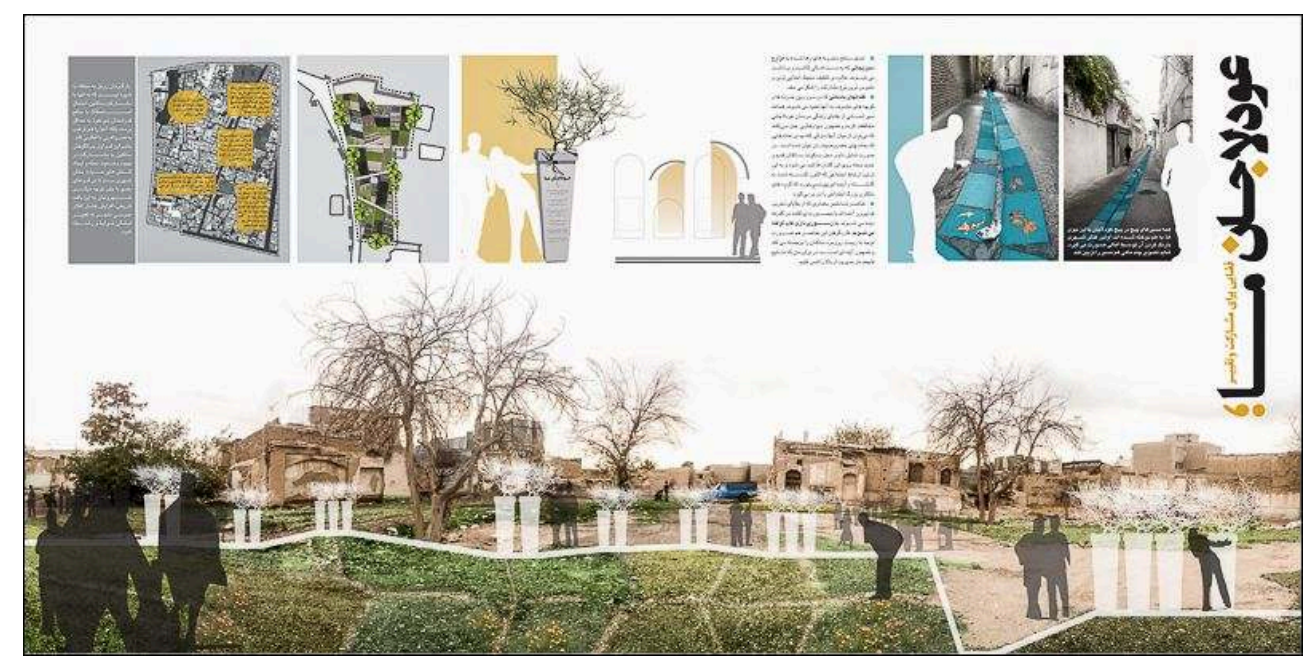

Proposition pour le concours portant sur le quartier d'Oudlajan, organisé par l'Institut des études urbaines de la ville de Téhéran.

Auteur : Niloufar Niksar, 2016.

19 En même temps, mon mari avait préparé quelques années avant un film sur les trottoirs de Téhéran et on s'intéressait à l'étude des caractéristiques des différents trottoirs dans les différents quartiers de Téhéran. Je faisais son travail de recherche documentaire. On a divisé Téhéran en plusieurs parties pour établir une classification des trottoirs. Quand on allait dans les quartiers centraux de Téhéran, les contacts étaient beaucoup plus faciles avec les gens et ils nous répondaient très facilement. Il y avait une échelle humaine, ils s'intéressaient au projet et ils étaient souvent ravis de s'asseoir et de nous parler de leurs problèmes. Dès qu'on montait dans la ville, dans les quartiers riches et plus modernes du nord de la ville, les gens étaient sceptiques et ne voulaient plus participer au projet.

20 On a compris que les habitants des quartiers anciens de Téhéran avaient un sens d'appartenance plus prononcé vis-à-vis de leur environnement de vie et ils parlaient beaucoup plus facilement de leur vie quotidienne. Ils nous posaient des questions sur 
notre projet et ils voulaient parfois qu'on intègre leurs problèmes à eux et qu'on en parle. À Oudlajan, on a parlé avec un habitant juif qui nous a fait visiter le quartier et les maisons anciennes qui étaient restées dans ce quartier. Ou par exemple, l'une des personnes avec qui nous avons beaucoup échangé était une dame qui était épicière. Alors que le quartier était connu pour son taux élevé de délinquance, elle avait tout à fait une autre expérience du quartier. Elle a parlé de son épicerie et de ses mobilités au quotidien dans le quartier et ses pratiques ne correspondaient pas tout à l'image stéréotypée du quartier. Mais en même temps, elle nous racontait qu'elle avait deux filles qu'elle n'arrivait pas à marier car à chaque fois qu'il y avait un candidat qui demandait leur main en mariage, ils pensaient qu'en habitant dans ce quartier, elles ne devraient pas être des filles bien et les familles ne donnaient plus suite. Ils pensaient qu'elles étaient peut-être droguées et c'est pour cela qu'elles habitaient encore dans ce quartier.

21 La mosquée de ce quartier était l'institution la plus populaire où les réunions de quartier avaient lieu. Dans nos enquêtes de terrain, on a saisi les rivalités qui peuvent exister entre les instances informelles comme les réunions à la mosquée et celles des autorités de la ville comme le conseil de la ville. Les membres du conseil de la ville et les habitants ne portaient pas le même regard sur la ville et son avenir. L'ensemble de ces expériences ont formé mon regard en tant qu'architecte et la nécessité de mettre ensemble le vécu des habitants à côté des demandes d'investisseurs. C'est seulement dans ce type de situation qu'on peut saisir les particularités de son environnement.

Il y avait un monsieur dans le quartier, par exemple, qu'on appelait «l'Inventeur »; il avait une vie sociale active et il arrivait à réunir les gens dans la mosquée. Il avait beaucoup de choses à dire et avait sa propre analyse des transformations urbaines dans ce quartier ; il nous disait par exemple : « cette maison a été achetée sous prétexte que c'était un lieu décadent où prenaient place des activités illégales alors qu'en réalité c'est $\mathrm{NAJA}^{7}$ qui l'a achetée ». Il avait fait une vraie recherche. Le jour où on a reçu le prix pour notre projet, on a demandé à ce qu'il vienne aussi à la rencontre qui a été organisée. Notre argument consistait à dire: «vous avez organisé une compétition pour le quartier d'Oudlajan mais il n'y pas un seul Oudlajani impliqué dans le projet, si vous trouvez une solution, on devrait aussi tenir compte de l'avis des habitants et non pas seulement avoir des gigolos (en français dans le texte, expression utilisée en persan pour désigner des gens branchés) du nord de Téhéran qui donnent leurs avis sans avoir aucune idée de ce qui se passe au quotidien ».

D'une certaine manière, nous les architectes, nous sommes souvent considérés comme un obstacle aussi bien pour les investisseurs que pour les usagers qui veulent se débarrasser de nous le plut tôt possible pour pouvoir aménager l'espace de la manière dont ils le souhaitent sans notre intervention. Grâce à l'expérience de l'écoute et de l'observation du vécu, j'avais envie de créer une vision d'entente entre les usagers et nous en tant que professionnels. C'était donc cela, notre approche pour mettre en place le projet Oudlajan : que l'architecte connaisse les usagers. L'interaction sociale avec les habitants d'Oudlajan a constitué le socle de ce projet.

- AM. Qui avait organisé la compétition?

- NN. C'est l'Institut des études urbaines de la ville de Téhéran, Tehran Institute ${ }^{8}$ un institut privé qui organise ce genre d'événement. Ils sont en relation avec le Conseil de la ville et un autre bureau d'architecture renommé et avaient aussi proposé le plan pour la zone $12^{9}$. Comme le thème de la participation est en même temps à la mode, ils 
en parlaient aussi dans leur discours. Ils avaient fait un blog en ligne et des journaux et ils essayaient de refléter les avis positifs des habitants sur les transformations du quartier. Mais il n'y avait aucune volonté d'informer les habitants sur les conséquences des projets qu'ils étaient en train de mettre en place dans ce quartier.

Notre point de vue était de dire qu'« avec les journaux et le web blog, vous recevez des réactions positives sur ce projet pour lui donner plus de légitimité mais vous n'acceptez aucune responsabilité des conséquences de ce type de projet». Par exemple, ils proposaient 300 millions de tomans (environ l'équivalent de 60000 euros à cette période) pour l'achat de leur maison mais avec cette somme, les habitants ne pouvaient vivre qu'à Parand, c'est-à-dire dans la banlieue sud de Téhéran.

Pour ces habitants qui ont toujours vécu dans le cœur de Téhéran, c'était presque impossible d'aller vivre dans une banlieue-dortoir. De nombreux habitants de ce quartier de Téhéran sont des ouvriers qui travaillent dans le grand bazar de Téhéran et, par conséquent, toute leur vie économique dépend de leur proximité vis-à-vis du Bazar, le poumon commerçant de la ville. Et qu'est-ce qui arriverait au bazar s'il perdait sa main-d'œuvre? En réalité le conflit était entre les gens du vieux bazar avec son fonctionnement traditionnel dans le tissu urbain et ceux du nouveau bazar, c'est-à-dire ceux qui cherchent à construire des centres commerciaux et des malls.

Dans ce jeu, les habitants n'avaient aucune place et bien sûr il était très difficile de faire accepter la part des habitants. Le plan qu'on proposait, on le savait, n'allait pas avoir beaucoup d'impact car cette vision n'est pas assez puissante face aux forces économiques décisives mais au moins on aurait marqué les esprits avec une nouvelle expérience et un autre regard sur l'environnement de vie et les conséquences d'un projet urbain. C'est la forme maximum d'agency que nous, dans l'environnement sociopolitique actuel de l'Iran, pouvons espérer avoir dans les projets.

29 En Iran, la question de la participation est liée à une série de lois juridiques très restrictives. La frontière entre la participation et l'infraction ou le délit est brouillée dans le contexte iranien. Donc en parlant de la participation, il est nécessaire aussi, en Iran, de connaître ses limites. Il y a un vrai besoin de juristes dans ce type d'activité. Par exemple, en travaillant sur les terrains vagues dans le centre de Téhéran, on était sûr que ces terrains avaient des propriétaires car il s'agissait de quartiers historiques avec des cadastres bien établis probablement depuis la période safavide (1501-17360. On pouvait faire de la végétation temporaire sur ces terrains mais on ne pouvait pas implanter des arbres qui auraient été des éléments permanents.

30 - AM. Sans vouloir entrer dans les clichés, est-ce que vous pensez qu'en tant que femme, l'exercice de votre métier représente des caractéristiques particulières dans le contexte iranien ? Je pose cette question aussi parce que vous avez écrit sur l'importance du rôle des femmes au foyer dans l'appropriation de l'espace intérieur et de leur importance pour les architectes.

31 - NN. On entend souvent dans notre métier ici que nous, les femmes qui travaillons dans ces métiers, nous sommes comme des hommes et nous nous comportons comme eux. Cette vision est en train de changer heureusement en Iran. C'est quand-même une société patriarcale et certaines femmes veulent reproduire le même type de pouvoir que les hommes au lieu de l'interroger et de proposer une autre manière de faire des choses. Certaines femmes, dans mon métier, reproduisent le même système de pouvoir que les hommes. C'est pour cela que je m'intéresse beaucoup aux femmes au foyer et que j'essaie toujours de prendre en compte leur quotidien dans la manière dont elles 
conçoivent leur espace intérieur. On entend souvent dire, dans notre métier, par mes collègues à propos de leur client: " Ah mais sa femme est venue dire ceci et cela et elle veut tout changer ». Alors que c'est elle qui va être la première à utiliser l'espace et donc elle a un droit.

Il y quelques années, j'ai eu la chance de faire la rencontre de clients de projets. Elles parlaient de leur besoin à la maison auxquels je n'avais pas forcément pensé. Quand je vais chez les gens, j'essaie de voir comment les femmes ont aménagé leur espace. Parfois, le plan architectural n'est pas terrible mais l'espace est accueillant. C'est toute la question, pour moi, de savoir ce qu'elles ont fait pour s'approprier l'espace car quand j'y entre je me sens mieux. Peut-être le fait d'être une femme m'aide à voir ces détails. La société moderne iranienne survalorise des femmes comme moi qui sommes dans le milieu professionnel et qui travaillons en dehors de la maison mais en réalité nous reproduisons souvent le même système de pouvoir que les hommes une fois entrées dans la vie professionnelle ou nous y contribuons alors que les petits actes des femmes au foyer sont très précieux et leur travail à la maison ne doit pas être ignoré. Le seul fait d'être en société n'est pas une valeur en soi et c'est pour ça que j'ai écrit cet article. - AM. Pour finir notre conversation, j'aimerais vous poser une question très générale pour connaître votre propre point de vue : à quel point pourrait-on associer, à votre avis, les problèmes socio-économiques de l'Iran contemporain à des questions urbaines ou à la manière dont l'architecture est exercée ? Comment et par quel moyen peut-on à votre avis regarder la société iranienne par la fenêtre de l'architecture et de l'urbanisme ? Je sais, c'est très général!

34 - NN. Comme je l'ai dit auparavant, l'économie iranienne est une économie malade avec de nombreux dysfonctionnements. Après la guerre Iran-Irak, ceci est devenu encore plus visible même si je pense que c'est dès les années 1960-1970 que l'économie iranienne a été affectée par de nouvelles formes de pathologies. En réalité, on a vu les effets secondaires plus tard et après la guerre Iran-Irak. La question du logement a toujours été très importante dans la période contemporaine et elle a souvent contribué au développement d'un système rentier dès les années 1970 avec l'arrivée des revenus pétroliers en Iran mais aussi après la guerre Iran-Irak.

En réalité, les politiques qui ont été mises en place après la guerre étaient très similaires à celles des années 1970 pendant la montée du prix du pétrole. La spéculation a marqué les projets de construction de logements et a marqué l'ensemble même du métier de l'architecture et la manière dont il est exercé. Les architectes et les urbanistes ont collaboré avec cette tendance et ils n'ont jamais remis en cause ce système. Je ne sais pas s'ils pouvaient faire autre chose mais en tout cas nous avons été un bon instrument au profit des grands réseaux économiques qui ont contribué à ce système rentier et aux affaires de spéculation autour du marché immobilier en Iran.

Pour nous, les architectes, la question de la modernité et de la modernisation de la ville a toujours constitué un objectif et une utopie et nous avons tous été emportés par ces idées de modernisation qui suivaient une logique capitaliste. En gros, toutes ces transformations, parfois néfastes, ont passé par notre canal, même si individuellement les architectes peuvent avoir de bonnes intentions. Il faut quand même rappeler qu'une grande partie des projets, disons environ 60 \%, n'ont même d'architecte et ce sont des constructeurs qui font tout le travail. Mais parfois la différence entre les projets qui ont eu des architectes et ceux qui n'en ont pas eu est seulement la façade et la dimension esthétique du projet. 


\section{NOTES}

1. Notamment dans les revues en persan d'architecture comme La revue d'architecture Hamshahri (http://memari.hmg.ir/Home/3160) et les revues d'art et de culture Tazarv et Filmkhaneh (http://filmkhanehmag.com/).

2. Pour plus d'information voir http://beatriceminda.de/iran-interrupted-2/\#1

3. Majid Rahnema (1924-2015) est un ancien diplomate et ministre iranien sous le régime Pahlavi .Il a représenté l'Iran à l'ONU de 1957 à 1971. Il travaille sur les problèmes de la pauvreté et les processus de production de la misère par l'économie de marché. En 1971, il a créé un Institut d'Études du Développement Endogène, inspiré par les idées éducatives de Paulo Freire, pour entamer un projet de développement de base avec les paysans de Lorestan. Après sa retraite en 1985, il a enseigné à l'Université de Californie à Berkeley durant six ans, puis, à partir de 1993, au Claremont Colleges de Pitzer. Il s'est établi ensuite en France, où il enseigne à l'Université Américaine de Paris ${ }^{4}$. Il est l'auteur de l'ouvrage paru en 2003 Quand la misère chasse la pauvreté (chez Fayard/Actes Sud).

4. Ce nom qui signifie littéralement «logement de l'affection » se réfère au projet de logements sociaux mis en place par l'ex-président Mahmoud Ahmadinejad au pouvoir de 2005 à 2013.

5. Oudlajan est un quartier historique de Téhéran, en Iran. Le quartier est entouré par la rue Pamenar, la rue Cyrus, Cheragh Bargh et la rue BozarJomehr. Oudlajan, en plus de Arg, Dolat, Sangelaj, Bazar et Chalmeidan, a constitué le Vieux Téhéran. Voir aussi Saidi, Mina (dir.), 2013. Le Téhéran des quartiers populaires. Transformation urbaine et société civile en République Islamique. Paris, Karthala/IFRI, $272 \mathrm{p}$.

6. Ayat Najafi est un réalisateur et scénariste iranien. Il a reçu de nombreux prix internationaux : au Festival international du film de Berlin et Outfest pour Football Under Cover; au Festival des films du monde de Montréal pour No Land's Song.

7. La Force de maintien de l'ordre de la République islamique d'Iran ou Force disciplinaire de la République islamique d'Iran, en abrégé NAJA, est la force de police en uniforme en Iran. La force a été créée au début de 1992 en fusionnant les Comités Shahrbani, Gendarmerie et Révolutionnaire Islamique en une seule force.

8. http://tehran-institute.com/

9. Un quartier central de Téhéran. 


\section{AUTEURS}

\section{NILOUFAR NIKSAR}

Niloufar Niksar est architecte à Téhéran et travaille sur des projets urbains et architecturaux variés. Diplômée de l'université Azad (Iran). En plus de ses activités d'architecte, elle a été scénographe au cinéma et au théâtre et a écrit de nombreux articles dans des revues d'art et d'architecture iraniennes.

\section{AMIN MOGHADAM}

Amin Moghadam est géographe au Centre d'études sur le golfe Persique et l'Iran à l'Université de Princeton aux Etats-Unis. Il a récemment publié :

- Moghadam A., Brones S. (dir.), 2016. Marchés et nouveaux territoires de l'art dans les villes du Sud, Géographie et Cultures, $\mathrm{n}^{\circ} 97$ [En ligne]. https://journals.openedition.org/gc/4248

- Moghadam A., Weber S., 2017. Circulating by default. Yerevan and Erbil, the backyards of Iranian mobility. In Vignal L. (dir.), Transnational Middle East. Places, People, Frontiers. Londres, Routledge, p. 164-181.

- Moghadam A., 2016. Tensions diplomatiques irano-arabes et pérennité de la présence des Iraniens à Dubaï. Confluences Méditerranée, 2016/2, N 97, p. 63-77. 